\title{
Charte de l'éthique des facultés de médecine
}

\author{
Conférence internationale des doyens et des facultés de médecine d'expression française (ADME)
}

\section{La charte de l'éthique des facultés de médecine s'ap- plique à deux domaines :}

- l'éthique de l'institution (dans ses activités de formation et de recherche) ;

- la formation initiale et continue à l'éthique médicale. La charte de l'éthique des facultés de médecine est un engagement moral que les facultés de médecine prennent entre elles. À cet égard, les responsables des facultés de médecine ou facultés des sciences de la santé réunies dans la CID M EF peuvent compter sur la solidarité et l'appui de la conférence et des autres facultés membres.

«Pour assurer sa pertinence, la charte est évaluée, explicitée et améliorée périodiquement ».

\section{Préambule}

Toutes les facultés de médecine fondent l'ensemble de leurs activités sur les valeurs qui inspirent le respect de la dignité de la personne humaine et la solidarité. Ces valeurs universelles ont vocation de régir les liens entre les acteurs du système de santé : malades, médecins, enseignants, chercheurs et étudiants. Elles imposent à chaque faculté le respect de ses engagements envers les étudiants qu'elle forme, envers les enseignants et chercheurs qui assurent sa dynamique et envers les institutions qui lui sont affiliées. Sa responsabilité envers la société requiert de chaque faculté d'optimiser la qualité des formations et des recherches qui sont organisées en son sein.

Chaque faculté de médecine se conforme aux textes législatifs et réglementaires en vigueur dans sa communauté nationale. Lorsque les activités dont elle est en charge ne font pas l'objet d'une codification juridique, ou que cette codification n'est pas suffisamment explicite, la faculté doit s'inspirer des recomman- dations des comités d'éthique nationaux et/ou internationaux. Dans tous les cas, les facultés de médecine fondent leurs activités sur le respect des $D$ roits de l'H omme.

\section{II - Les missions des facultés de médecine}

Chaque faculté définit ses objectifs institutionnels : ils sont adaptés à la société afin qu'ils répondent aux besoins de santé de la population et des individus qui la composent.

Le but de l'enseignement dans les facultés de médecine est de former un médecin responsable, c'est-à-dire un homme de science à l'écoute et au service d'une population, capable de prendre soin de la santé individuelle et collective des personnes qui se confient à lui ou que la société lui confie. À cet effet, chaque faculté cherche à concilier les réponses aux besoins de santé de chacun et les exigences de répartition équitable entre tous des ressources sanitaires disponibles. D e ce fait, la formation initiale et continue à l'éthique médicale se situe parmi les priorités éducatives de la faculté.

Le but de la recherche faite en faculté de médecine est la production de savoirs pour le bénéfice individuel immédiat ou potentiel des patients et/ou un bénéfice collectif, qui tiennent compte des répercussions sur l'environnement, les individus et la société.

Pour ses prestation s de services, chaque faculté de médecine a un partenariat avec les autres acteurs du système de santé ; l'objectif est d'assurer le meilleur niveau de santé pour l'ensemble des citoyens en respectant un esprit d'équité et une utilisation optimale des moyens.

D e ces principes inspirant les missions et prestations des facultés de médecine, il découle que la finalité de la formation médicale et de la recherche médicale ne peut être essentiellement d'ordre lucratif.

De ces mêmes principes, il ressort que les facultés de médecine assurent l'évaluation de leurs activités. 


\section{III - Les personnes ressources des facultés de} médecine pour l'enseignement et la recherche

Les critères de sélection des personnes ressources sont les compétences professionnelles spécifiques à chaque fonction dans le respect des objectifs institutionnels et en accord avec les buts assignés à la formation médicale tels qu'ils ont été définis dans la faculté. Le recrutement et la promotion des personnes ressources en enseignement, en recherche, en soutien technique et administratif par la faculté de médecine se font sur des critères et sel on des méthodes équitables et transparentes, sans discrimination liée à la race, au sexe, à l'âge, à l'appartenance socio-économique, politique ou religieuse.

Tous les enseignants et/ou chercheurs, ainsi que les personnes de soutien technique et administratif doivent accepter le principe du regard et d'appréciation éthique d'un tiers pour tout ce qu'ils entreprennent de faire dans leurs missions.

\section{IV - L'éthique de l'apprentissage des étudiants en médecine}

\section{Concernant les étudiants en médecine :}

l'entrée et la sélection durant les études à la faculté de médecine se font sur des critères et selon des méthodes équitables, sans discrimination liée à la race, au sexe, à I'appartenance socioéconomique, politique ou religieuse. Les seuls critères retenus sont en accord avec les buts inhérents à la formation médicale tels qu'ils ont été définis et avec les capacités individuelles de l'étudiant.

La faculté de médecine assure à tous les étudiants en formation initiale et à tous les praticiens en formation continue l'équité de l'accès aux moyens d'apprentissage dans le respect des objectifs qu'elle a fixés et en optimisant les moyens dont elle dispose.

\section{Concernant la participation des malades à l'ensei- gnement médical :}

la formation initiale et continue des médecins et autres professionnels de santé est un processus d'acquisition de compétences professionnelles. Cette formation requiert la pratique. De ce fait, les médecins et autres professionnels de santé ont besoin de la participation des malades à leur formation. Ces malades peuvent être invités à la faculté; ils peuvent être sollicités durant leur séjour hospitalo-universitaire ou dans d'autres structures hospitalières ou extra - hospitalières.
D e ce fait, dans les ententes qui les lient aux institutions de soins, les facultés de médecine conviennent des procédures qui assurent le respect de la dignité et le consentement des personnes malades participant à l'enseignement.

\section{Concernant l'organisation des études et des stages :} chaque faculté de médecine doit promouvoir pour elle même et avec les institutions partenaires des recommandations et des procédures visant la qualité éthique des études et des stages en accord avec le but de la formation médicale. Elle doit garantir la qualité éthique des formations dont elle est directement responsable.

\section{Concernant les savoirs enseignés :}

chaque faculté de médecine est responsable de la dimension éthique de l'utilisation des savoirs qui sont dispensés au cours de ses programmes d'études : ces savoirs enseignés ne sont pas obligatoirement neutres au regard de ce qu'ils impliquent : dans toutes leurs dimensions, ils doivent impérativement respecter la dignité de la personne humaine.

\section{V - L'éthique de la recherche en faculté de médecine}

Chaque faculté de médecine adosse sa recherche universitaire aux principes de l'humanisme et de la qualité scientifique.

\section{Concernant les étudiants participant aux recherches :}

les étudiants agissant comme des personnes ressources pour la recherche doivent bénéficier de la même considération que les autres personnels de recherche, qu'il s'agisse d'une part, des conditions général es de travail et d'autre part, des critères de sélection et d'évaluation.

\section{Concernant les laboratoires de recherche des facultés de médecine :}

les thématiques et les méthodes sont choisies selon les meilleurs effets escomptés et selon les retombées les plus bénéfiques, autant pour les personnes sujets de recherches que pour la population. Ces choix ne peuvent justifier la perte d'indépendance professionnelle des chercheurs ou de la faculté de médecine elle-même. Ces choix impliquent le respect de la dignité de la personne humaine.

L'analyse et la divulgation des résultats de recherche 
assurent la protection des personnes concernées selon le principe de la confidentialité médicale.

Par ses activités de recherche, chaque chercheur reliéà la faculté contribue au renom de sa structure propre ainsi qu'à celui de l'institution ellemême, dans le respect des autres chercheurs.

\section{Concernant les malades participant à la recherche :} les médecins et autres professionnels de santé ont parfois besoin de la participation de personnes malades à leurs recherches. Dans les ententes qui les lient aux malades et aux institutions de soins, les facultés de médecine conviennent des procédures qui assurent le respect de la dignité et l'autonomie des malades participant à la recherche en tenant compte des principes de bienfaisance. Ce même respect de la dignité de la personne humaine est exigé lors de la participation de volontaires sains. $D$ es règles d'éthique rigoureuses doivent être appliquées lors de dons de corps, d'organes et de produits humains en post mortem pour la recherche ou l' enseignement ; elles respectent dans tous les cas la volonté des donneurs quand elle a été exprimée et les lois en vigueur dans le pays.

\section{Concernant l'utilisation et la valorisation des savoirs issus de la recherche :}

les facultés de médecine sont responsables de la dimension éthique des savoirs issus des recherches menées sous leur égide. II est rappeléque la production de savoirs par la recherche sous l'égide des facultés de médecine a pour but un bénéfice individuel immédiat ou potentiel des patients et/ou un bénéfice collectif qui tiennent compte des répercussions éventuelles sur l'environnement, les individus et la société.

Les savoirs issus des travaux de recherche ne sont pas obligatoirement neutres et ont un aspect éthique dont les investigateurs sont appelés par les facultés de médecine à prendre la mesure. Leur utilisation ne peut être considérée éthique que si elle respecte certains critères dont le principal est le respect de la dignité de la personne humaine.

Les retombées et bénéfices éventuels doivent profiter à tous les acteurs de la recherche au premier rang desquels il y a les malades concernés.

Tous les chercheurs y compris les étudiants doivent profiter des retombées scientifiques à proportion de leur participation active aux travaux, à la condition que cela n'entache pas leur indépendance professionnelle, que ce soit dans leurs activités de soins, de recherche ou d'enseignement. Les facultés doivent assurer aux chercheurs, y compris les étudiants impliqués, la reconnaissance de leur propriété intellectuelle.

\section{$\mathrm{VI}$ - L'éthique de la coopération des facul- tés de médecine}

La coopération concernant l'enseignement comme la recherche est mise en œuvre avec des institutions dans des pays pouvant avoir des niveaux de développement différents. Dans ces ententes de coopération, chaque faculté de médecine reconnaît les mêmes valeurs centrées sur la dignité humaine, applique les mêmes critères centrés sur le principe de justice et respecte les mêmes engagements de transparence et de responsabilité que dans ses ententes nationales ou régionales.

\section{VII - L'apprentissage de l'éthique médicale}

Chaque faculté de médecine a un programme de formation explicite au professionnalisme médical, lequel inclut la formation initiale et continueà l'éthique médicale. 


\section{Concepts et Innovations}

\section{Annexe 1: \\ Comité de la charte de l'éthique des facultés de médecine}

\section{Coordonnateur :}

Pr Jacques-H enri BARRIER, Président du Conseil Pédagogique de la CID M EF, N antes (France)

\section{Secrétaire :}

Pr Lucie BRAZEAU -LAM ONTAGN E, Professeure titulaire de Radiologie; Présidente de l' Association des professeures et professeurs de la Faculté de M édecine de l'U niversité de Sherbrooke (APPFM US)

\section{Ont coopéré:}

Pr M ario ALVAREZ, D oyen de la Faculté de M édecine d'Etat de Port au Prince (H aïti)

Pr N ourredine BACHIR-BOUIADJRA, D oyen de la Faculté de M édecine d'O ran (Algérie)

$\operatorname{Pr}$ G eneviève BARRIER-JACOB, ancien membre du Comité Consultatif National d' Ethique de France

(Paris, France)

Pr Abdelmajid BELM AHI, ancien D oyen de la Faculté de M édecine de Rabat (M aroc),

ancien M inistre de la Santé, Président de la CID M EF

Pr Abdelatif BEN M ATI, D oyen de la Faculté de M édecine de Constantine (Algérie)

Dr Charles BOELEN, Consultant international en systèmes et personnels de santé; ancien coordonnateur du programme de l'O M S des ressources humaines pour la Santé (Genève, Suisse)

Pr André BO N N IN , Université M édicale Virtuelle Francophone (Paris, France)

Pr Bernard CH ARPEN TIER, Président de la Conférence des D oyens des Facultés de M édecine Françaises, ler Vice-Président de la CID M EF (Paris, France)

Pr Raymond CO LIN , ancien Vice D oyen aux études de la Faculté de M édecine de Rouen (France)

Pr Luc COTE, professeur titulaire de médecine familiale et directeur du Centre de développement pédagogique de la Faculté de M édecine del'U niversité Laval (Q uébec, C anada)

Pr G érard DABO UIS, service d'oncologie médicale et de soins palliatifs, CHU de Nantes (France)

Pr M iche DETILLEUX, Conseil National de l'O rdre des M édecins de France (Paris, France)

Pr Somian F. EH UA, chirurgien, responsable de la cellule pédagogique de la Faculté de M édecine d'Abidjan (Côte d' Ivoire)

Pr Sahloul A. ESSO U SSI, ancien D oyen de la Faculté de M édecine de Sousse (Tunisie)

Pr Pierre FARAH, ancien D oyen de la Faculté de M édecine St Joseph (Beyrouth, Liban), ancien Président delaCIDMEF

Pr Jean-Paul FRAN C KE, Secrétaire G énéral de la Conférence des $D$ oyens des Facultés de M édecine de France, Doyen de la Faculté de médecine de Lille (France)

Pr Jacques FREN ET TE, M édecin Généraliste, Vice-D oyen de la Faculté de M édecine de l'U niversité Laval (Q uébec, C anada)

Pr Abdelaziz GH ACH EM , ancien D oyen de la Faculté de M édecine de Tunis (Tunisie)

Pr Bernard GAY, ancien Président du Collège $N$ ational des $G$ énéralistes Enseignants de France (Bordeaux, France)

Pr Roger GIL, D oyen de la Faculté de M édecine de Poitiers (France)

Pr André G O U AZE, Président Fondateur, Directeur G énéral de la CID M EF (Tours, France)

Pr Roger GUILLEM IN , Prix N obel de M édecine, San D iego La Jolla (Etats-U nis)

Pr Claude H URIET, Sénateur honoraire, membre du C omité International de bioéthique de I'U N ESCO

(N ancy, France)

Pr François JACO B, Prix N obel de médecine (Paris, France)

Pr François LEM AIRE, réanimateur, Président de la Fondation M aurice Rapin (Paris, France) 
Pr Guy LLO RCA, Professeur deThérapeutique, Président du Comité d'éthique du C.H .U . de Lyon (France) et membre de l'O bservatoire $\mathrm{N}$ ational d'Ethique C linique

Pr H ubert M ARCOUX, Président du Comité D épartemental de l'enseignement de l' éthique en médecine familiale, Faculté de M édecine de I'U niversité L aval à $Q$ uébec ( $C$ anada)

Pr Badie A. MEH ADJI, D oyen de la Faculté de M édecine et de Pharmacie de $M$ arrakech ( $M$ aroc)

Pr Petru M IRCEA, Charge de mission à la CID M EF ; ancien D oyen de la Faculté de M édecine de Cluj-N apoca (Roumanie)

Pr Didier M OU LIN, ancien Doyen de la Faculté de M édecine de l' U niversité Catholique de Louvain (Belgique)

Pr Koffi G. N 'D AKEN A, Chef service Radiologie CHU Tokoin-Campus, D oyen dela Faculté M ixte de $M$ édecine et de Pharmacie Université de Lomé (Togo)

Dr Jacques N D EM AN GA KAM O U N E, urologue, ancien D oyen de la Faculté des Sciences de la Santé (Bangui, République Centrafricaine)

Pr M oïse O LIVEIRA, ancien Recteur de l' Université de Libreville (G abon)

Pr Rambré M . O UIM IN GA, ancien Secrétaire G énéral du CAM ES, M embre du Conseil National d' Ethique (O uagadougou, Burkina-Faso)

Pr H élène PELLET, ancien D oyen de la Faculté de M édecine de Lyon Grange Blanche (France)

Pr Pierre POTVIN , ancien D oyen de la Faculté de M édecine de I'U niversité Laval à Q uébec (C anada) ;

Président du Conseil d' Evaluation de la CID MEF

Pr Pascal RAKOTOBE, Recteur de I'U niversité d' Antananarivo (M adagascar)

Pr Jacques RO LAND, ancien Président de la Conférence Française des D oyens des Facultés de $M$ édecine, membre du Conseil N ational de l'O rdre des M édecins ( $N$ ancy, France)

Pr Jean-Claude RO LLAN D, ancien D oyen de la Faculté de M édecine de Tours ; (France)

Directeur Général Adjoint de la CIDM EF

Pr Jean-Jacques RO M BO UTS, D oyen de la Faculté de M édecine de I'U niversité C atholique de Louvain

(Belgique)

Pr M arcel RO OZE, ancien Doyen de la Faculté de M édecine de I'U niversité Libre de Bruxelles (Belgique)

Pr Didier SICARD, Président du C omité C onsultatif N ational d'Ethique de France (Paris, France)

Pr M aurice SO SSO, ancien Vice Recteur de I'U niversité de Yaoundé (C ameroun)

M r Jean-Philippe SZYLOW ICZ, Ingénieur, ancien enseignant à l'Ecole Supérieure d' Eletricité Paris (France)

Pr N awar TEW FIK, Faculté de M édecine de I'U niversité de Sherbrooke

PrT ÔN THÂT BACH, ancien doyen de la Faculté de M édecine, ancien Recteur de l'U niversité

(H anoï, Viet-Nam)

Pr M oussa TRAORÉ, Président de la Conférence Africaine des D oyens des Facultés de M édecine d'Expression

Française (CAD M EF) (Bamako, M ali)

Pr D ominique VAN PEE, Université Catholique de Louvain (Belgique)

M r G ilbert VICEN TE, Secrétaire Général de la Faculté de M édecine de Strasbourg (France),

Président del' AUFEM 0

Pr Patrick VIN AY, ancien D oyen de la Faculté de M édecine de M ontréal (Canada),

ancien Président de la CIDM EF 


\section{Concepts et Innovations}

\section{Annexe 2 : \\ M éthode de rédaction de la charte}

La décision de la rédaction d'une charte de l'éthique des facultés de médecine a été prise lors des réunions du bureau permanent et de la session plénière de la CID M EF àTours en mai 2001, après avoir entendu le rapport d'un atelier animé par J-H. Barrier ( $\mathrm{N}$ antes), A. Ghachem (Tunis) et M. O liverra (Libreville) et composé de N. Bachir-Bouiadjra (Oran Algerie), A. Belmahi (Rabat Maroc), P. Chedid (Beyrouth, Liban), L. Degail (IN SERM Paris, France), J.-F. D enef (Louvain, Belgique), J. Fourcade (M ontpellier, France), A. M aaouni (Fes, M aroc), B.-A M ehadji (M arrakech, M aroc), D. M oulin (Louvain, Belgique), J. N 'D emanga Kamoune (Bangui, Centrafrique), P. N 'D umbe (Yaounde, C ameroun), D . Thiam (D akar, Sénégal) .

Un comité a alors été constitué (f liste supra) et a travaillé pendant 18 mois: une liste de discussion électronique a permis les échanges. Chaque proposition nouvellea été intégrée dans le texte puis soumise à la discussion géné rale. La coordination de ce groupe de travail a été assurée par J. Barrier ( $N$ antes) et le secrétariat par L. BrazeauLamontagne (Sherbrooke).

Les premières propositions du comité ont été revues par la CID M EF (session plénière de Phnom Penh en février 2003) à l'issue d'un atelier auquel participaient : Pr J.-H . Barrier ( $N$ antes, France), coordonnateur de la charte, animateur del'atelier, et Pr A. Benmati, D oyen de la Faculté de Constantine, Algérie, rapporteur. Les D oyens ou leurs représentants : F.-F. Angwafo (Yaounde, Cameroun), A. Cucu (Brasov, Roumanie), J.-L. D ebru (Grenoble, France), Y. Djanhan (Bouaké, Côte d' Ivoire), I. Georgescu (Craiova, Roumanie), R. Gil (Poitiers, France), V. Gliga (Tirgu-M ures, Roumanie), BA M ehadji ( $M$ arrakech, Maroc) N M iu (Cjuj-N apoca, Roumanie), SA O uld M ogueya (N ouakchott, M auritanie), G.K N 'D akena (Lome, Togo), J. N demanga-Kamoune (Bangui, RCA), A. N yakayiro (Butare, Rwanda), J.-J. Rombouts (Louvain, Belgique), D. Thiam (D akar, Sénégal), Thouvisouk Bounsai (Vientiane, Laos), Tôn Thât Bach ( $\mathrm{H}$ anoï, Viet-Nam). Autres participants : R.-M . O uiminga (O uagadougou, Burkina-Faso) Conseiller Permanent de la CID M EF et ancien Secrétaire G énéral du CAMES, C. Binet (Tours, France) Chargé de mission Technologies éducatives CIDMEF et G. Vicente (Strasbourg, France) Président de l' AUFEM O

Le comité a repris ses échanges pendant quelques mois. Les propositions intermédiaires ont été exposées par J.-H.Barrier en plénière lors des 15 èmes journées universitaires francophones de pédagogie médicale de $N$ ancy (22-25 avril 2003). La problématique de l'apprentissage de l'éthique a fait l'objet d'un large examen de la collectivité enseignante francophone à plusieurs niveaux :

1- Les résultats d'une enquête du Conseil pédagogique de la CID M EF sur l'apprentissage de l'éthique dans les facultés de médecine francophones ont été rapportés par $\mathrm{G}$. Llorca.

2- Un atelier de formation (L. Brazeau-Lamontagne et J.-H . Barrier) sur l'apprentissage du rai sonnement éthique (apprentissage de la démarche éthique ou comment acquérir des compétences éthiques?).

3- Un atelier stratégique, destiné aux responsables des facultés, sur l' implantation d'un programme de formation à la professionnalisation des futurs médecins (de l'éthique à la relation médecin-patient) a réuni une centaine de participants (experts : J.-H . Barrier, L. Brazeau-Lamontagne, G. Llorca).

4- Une session de communications libres a permis d'échanger les expériences dans une vingtaine de facultés de médecine du monde francophone (citons : Strasbourg, Dijon, N antes, Angers, Lille, Brest, Louvain, M ontréal, Beyrouth, etc.).

Les 12 mois suivants ont étél'occasion pour le comité de finaliser la charte avant proposition pour validation par le Bureau Permanent de la CID M EF à Bordeaux en mai 2004. 


\section{Annexe 3 : \\ D ocuments et références utilisés par le comité de la charte}

- Serment d' H ippocrate.

- Déclaration universelle des droits de l'homme.

- Convention internationale des droits del'Enfant. Résolution 44/25 adoptée par l'Assembléegénérale del'O NU le 20 N ovembre 1989.

- D éclaration universelle sur le génome humain et les droits de l'homme. Adoptée à la $29^{\circ}$ session de la conférence générale del'U N ESCO le 11 novembre 1997.

- Déclaration des droits de l'homme et du citoyen. S Rials Ed. Paris : H achette 1988.

- Association M édicale Mondiale :

- Serment de Genève de l'Association médicale mondiale:

- Déclaration d'H elsinki

- $51^{\text {me }}$ Assemblée générale de l'Association médicale mondiale (octobre 1999) sur l'inclusion de l'éthique médicale et des droits de l'homme dans les programmes des écoles de médecine

- O rganisation M ondiale de la Santé (OM S) :

- D eveloping protocols for change in medical education. Ch. Boelen, J. Des M archais, CW. D ohner, M P. Kantrowitz O M S 1992, 1.

- M édecins pour la santé. Une stratégie mondiale de l'O M S pour la réorientation de l'enseignement de la médecine et de la pratique médicale en faveur de la santé pour tous. O rganisation M ondiale de la Santé, Genève, Suisse, 1996. (réf.: WH O / H RH /96.1 ).

- Définir et mesurer la responsabilité sociale des facultés de médecine. $C h$. Boelen, J. H eck. O rganisation M ondiale de la Santé, Genève, Suisse, 2000 (réf.: WHO / HRH / 95.7 ).

- Universities and the health of the disadvantaged. Eds. DS Blumenthal et Ch. Boelen. O M S 2000.

- Rapport des Journées d'études internationales de l'O M S et de la CID M EF : «La Faculté de médecine et le médecin praticien du XXI ème siècle », Bruxelles 9-12 avril 1996, I. Pelc et L. Cassiers, Université Catholique de Louvain, Université Libre de Bruxelles, Fondation pour l'éude et la prévention des maladies de civilisation, Ed. Labor Bruxelles, 1998.

- Project of the ABIM Foundation, ACP-ASIM Foundation, and European Federation of Internal M ediane. M edical professionalism in the new milleni um: a physician' charter. Ann Intern M ed 2002; 163: 243-246 ; traduction dans Pédagogie M édicale $2004 ; 5$ : 43-45.

- Université Laval (Q uébec-C anada) :

- D éclaration des droits des étudiants et des étudiantes.

- Règlement sur leharcèlement sexuel.

- Code de N uremberg.

- Comité Consultatif $N$ ational d'Ethique de France pour les sciences dela vie et de la Santé: la coopération dans le domaine de la recherche biomédicale entre équipes françaises et équipes de pays en voie de développement économique $N^{\circ} 41$ (18 décembre 1993). Actualisation dans l'avis $n^{\circ} 78$ : «Inégalités d'accès aux soins et dans la participation à la recherche à l'échelle mondiale - progrès éthiques » du 18 septembre 2003.

- D éclaration d'Abidjan (conférence des ministres de l'enseignement supérieur et de la recherche des états et gouvernements ayant le français en partage), AU PELF-U REF Abidjan décembre 1993.

- Ethique et professions de santé Rapport au ministre de la santé, de la famille et des personnes handicapées. A Cordier, Paris M ai 2003

- La dignité de l'H omme Actes du Colloque de la Sorbonne, Paris. Novembre 1992. Ed. P. M agnard, Paris Champion 1995.

- De la dignité humaine. T de Koninck, Paris: PUF, 1995.

- Professionnalisme et délibération éthique. GA. Legault, Presses de I'U niversité du Q uébec, 1999. 